\title{
Bifurcation and Asymptotics for Elliptic Problems with Singular Nonlinearity
}

\author{
Vicenţiu Rădulescu \\ A mon Maître, avec reconnaissance
}

\begin{abstract}
We report on some recent existence and uniqueness results for elliptic equations subject to Dirichlet boundary condition and involving a singular nonlinearity. We take into account the following types of problems: (i) singular problems with sublinear nonlinearity and two parameters; (ii) combined effects of asymptotically linear and singular nonlinearities in bifurcation problems; (iii) bifurcation for a class of singular elliptic problems with subquadratic convection term. In some concrete situations we also establish the asymptotic behaviour of the solution around the bifurcation point. Our analysis relies on the maximum principle for elliptic equations combined with adequate estimates.
\end{abstract}

Mathematics Subject Classification (2000). Primary 35J60; Secondary 35B32, 35B40.

Keywords. Singular nonlinearity, bifurcation, asymptotic analysis, maximum principle.

\section{Motivation and Previous Results}

I will report on some results contained in our recent papers [3, 7, 8, 9, 10, 11, that are closely related to the study of some problems on blow-up boundary solutions. More precisely, consider the elementary example

$$
\begin{cases}\Delta u=u^{p} & \text { in } \Omega, \\ u>0 & \text { in } \Omega, \\ u=+\infty & \text { on } \partial \Omega,\end{cases}
$$

Partially supported by Grant 12/2004 with the Romanian Academy. 
where $\Omega \subset \mathbb{R}^{N}$ is a smooth bounded domain and $p>1$. Then the function $v=u^{-1}$ satisfies

$$
\begin{cases}-\Delta v=v^{2-p}-\frac{2}{v}|\nabla v|^{2} & \text { in } \Omega, \\ v>0 & \text { in } \Omega, \\ v=0 & \text { on } \partial \Omega .\end{cases}
$$

The above equation contains both singular nonlinearities (like $v^{-1}$ or $v^{2-p}$, if $p>2$ ) and a convection term (denoted by $|\nabla v|^{2}$ ). These nonlinearities make more difficult to handle problems like (1.1). Our purpose in this paper is to give an overview on some old and new results in this direction. We recall the pioneering paper [5] that contains one of the first existence results for singular elliptic problems. In fact, it is proved in [5] that the boundary value problem

$$
\begin{cases}-\Delta u-u^{-\alpha}=-u & \text { in } \Omega, \\ u>0 & \text { in } \Omega, \\ u=0 & \text { on } \partial \Omega\end{cases}
$$

has a solution, for any $\alpha>0$. Let us now consider the problem

$$
\begin{cases}-\Delta u-u^{-\alpha}=\lambda u^{p} & \text { in } \Omega, \\ u>0 & \text { in } \Omega, \\ u=0 & \text { on } \partial \Omega,\end{cases}
$$

where $\lambda \geq 0$ and $\alpha, p \in(0,1)$. In 4 it is proved that problem (1.2) has at least one solution for all $\lambda \geq 0$ and $0<p<1$. Moreover, if $p \geq 1$, then there exists $\lambda^{*}$ such that problem (1.2) has a solution for $\lambda \in\left[0, \lambda^{*}\right)$ and no solution for $\lambda>\lambda^{*}$. In [4] it is also proved a related non-existence result. More exactly, the problem

$$
\begin{cases}-\Delta u+u^{-\alpha}=u & \text { in } \Omega, \\ u>0 & \text { in } \Omega, \\ u=0 & \text { on } \partial \Omega\end{cases}
$$

has no solution, provided that $0<\alpha<1$ and $\lambda_{1} \geq 1$ (that is, if $\Omega$ is "small"), where $\lambda_{1}$ denotes the first eigenvalue of $(-\Delta)$ in $H_{0}^{1}(\Omega)$.

Problems related to multiplicity and uniqueness become difficult even in simple cases. In [16] it is studied the existence of radial symmetric solutions to the problem

$$
\begin{cases}\Delta u+\lambda\left(u^{p}-u^{-\alpha}\right)=0 & \text { in } B_{1}, \\ u>0 & \text { in } B_{1}, \\ u=0 & \text { on } \partial B_{1},\end{cases}
$$

where $\alpha>0,0<p<1, \lambda>0$, and $B_{1}$ is the unit ball in $\mathbb{R}^{N}$. Using a bifurcation theorem of Crandall and Rabinowitz, it has been shown in 16 that there exists $\lambda_{1}>\lambda_{0}>0$ such that the above problem has no solutions for $\lambda<\lambda_{0}$, exactly one solution for $\lambda=\lambda_{0}$ or $\lambda>\lambda_{1}$, and two solutions for $\lambda_{0}<\lambda \leq \lambda_{1}$.

Our purpose in this survey paper is to present various existence, and nonexistence results for several classes of singular elliptic problems. We also take into account bifurcation nonlinear problems and establish the precise rate decay of the 
solution in some concrete situations. We intend to reflect the "competition" between different quantities, such as: sublinear or superlinear nonlinearities, singular nonlinear terms (like $u^{-\alpha}$, for $\alpha>0$ ), convection nonlinearities (like $|\nabla u|^{q}$, with $0<q \leq 2)$, as well as sign-changing potentials.

\section{A Singular Problem with Sublinear Nonlinearity}

Consider the following boundary value problem with two parameters:

$$
\begin{cases}-\Delta u+K(x) g(u)=\lambda f(x, u)+\mu h(x) & \text { in } \Omega, \\ u>0 & \text { in } \Omega, \\ u=0 & \text { on } \partial \Omega\end{cases}
$$

where $\Omega$ is a smooth bounded domain in $\mathbb{R}^{N}(N \geq 2), K, h \in C^{0, \gamma}(\bar{\Omega})$, with $h>0$ on $\Omega$, and $\lambda, \mu$ are positive real numbers. We suppose that $f: \bar{\Omega} \times[0, \infty) \rightarrow[0, \infty)$ is a Hölder continuous function which is positive on $\bar{\Omega} \times(0, \infty)$. We also assume that $f$ is non-decreasing with respect to the second variable and is sublinear, that is,

$$
\begin{aligned}
& (f 1) \quad \text { the mapping }(0, \infty) \ni s \longmapsto \frac{f(x, s)}{s} \text { is non-increasing for all } x \in \bar{\Omega} \text {; } \\
& (f 2) \quad \lim _{s \downarrow 0} \frac{f(x, s)}{s}=+\infty \text { and } \lim _{s \rightarrow \infty} \frac{f(x, s)}{s}=0, \text { uniformly for } x \in \bar{\Omega} .
\end{aligned}
$$

We assume that $g \in C^{0, \gamma}(0, \infty)$ is a non-negative and non-increasing function. A fundamental role in our analysis will be played by the numbers

$$
K^{*}:=\max _{x \in \bar{\Omega}} K(x), \quad K_{*}=\min _{x \in \bar{\Omega}} K(x) .
$$

Our first theorem is a non-existence result and it concerns nonlinearities with strong blow-up rate at the origin (like $u^{-\alpha}$, with $\alpha \geq 1$ ).

Theorem 2.1. Assume that $K_{*}>0$ and $f$ satisfies $(f 1)-(f 2)$. If $\int_{0}^{1} g(s) d s=+\infty$, then problem (2.1) has no classical solution, for any $\lambda, \mu>0$.

Next, we assume that the growth of the nonlinearity is described by the following conditions:

$$
\lim _{s \downarrow 0} g(s)=+\infty
$$

(g2) there exist $C, \delta_{0}>0$ and $\alpha \in(0,1)$ such that $g(s) \leq C s^{-\alpha}$, for all $s \in\left(0, \delta_{0}\right)$.

The above conditions $(g 1)$ and $(g 2)$ are fulfilled by singular nonlinearities like $g(u)=u^{-\alpha}$, with $\alpha \in(0,1)$. Obviously, hypothesis $(g 2)$ implies the following Keller-Osserman type condition around the origin:

(g3) $\quad \int_{0}^{1}\left(\int_{0}^{t} g(s) d s\right)^{-1 / 2} d t<\infty$.

As proved by Bénilan, Brezis and Crandall [1], condition ( $g 3)$ is equivalent to the property of compact support, that is, for every $h \in L^{1}\left(\mathbb{R}^{N}\right)$ with compact 
support, there exists a unique $u \in W^{1,1}\left(\mathbb{R}^{N}\right)$ with compact support such that $\Delta u \in L^{1}\left(\mathbb{R}^{N}\right)$ and $-\Delta u+g(u)=h$, a.e. in $\mathbb{R}^{N}$. That it is why it is natural to try to find solutions in the class

$$
\mathcal{E}=\left\{u \in C^{2}(\Omega) \cap C(\bar{\Omega}) ; \Delta u \in L^{1}(\Omega)\right\} .
$$

In the case where the potential $K(x)$ has a constant sign, the following results hold.

Theorem 2.2. Assume that $K_{*}>0, f$ satisfies $(f 1)-(f 2)$, and $g$ satisfies $(g 1)-$ (g2). Then there exists $\lambda_{*}, \mu_{*}>0$ such that:

- problem (2.1) has at least one solution in $\mathcal{E}$ either if $\lambda>\lambda_{*}$ or if $\mu>\mu_{*}$.

- problem (2.1) has no solution in $\mathcal{E}$ if $\lambda<\lambda_{*}$ and $\mu<\mu_{*}$.

Moreover, if either $\lambda>\lambda_{*}$ or if $\mu>\mu_{*}$, then problem (2.1) has a maximal solution in $\mathcal{E}$ which is increasing with respect to $\lambda$ and $\mu$.

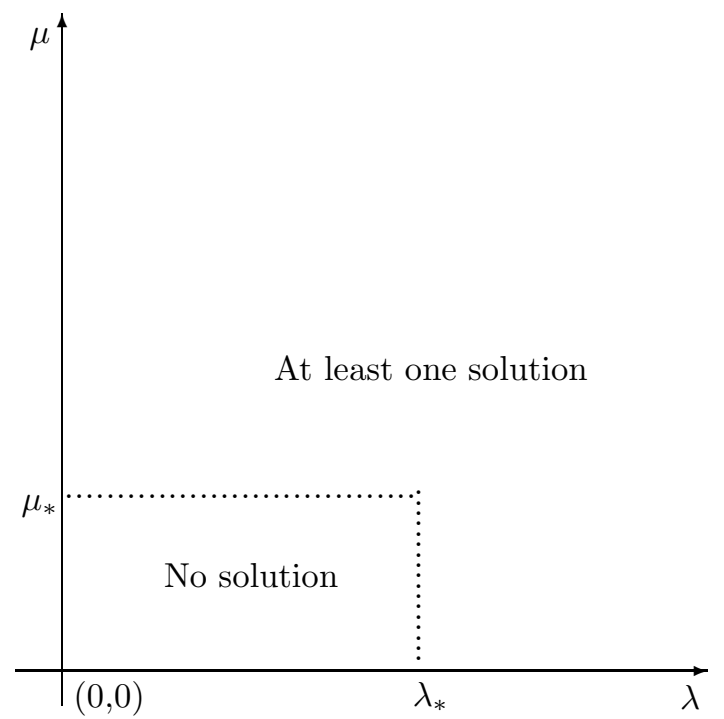

Figure 1. The dependence on $\lambda$ and $\mu$ in Theorem 2.2

At this stage we are not able to describe the behaviour in the following cases: (i) $\left[\lambda=\lambda_{*}\right.$ and $\left.0<\mu \leq \mu_{*}\right]$ and (ii) $\left[0<\lambda \leq \lambda_{*}\right.$ and $\left.\mu=\mu_{*}\right]$. We conjecture that existence or non-existence results can be established in conjunction with a more precise description of the decay rate of the potential coefficients and nonlinearities.

Theorem 2.3. Assume that $K^{*} \leq 0, f$ satisfies conditions $(f 1)-(f 2)$ and $g$ satisfies $(g 1)-(g 2)$. Then problem (2.1) has a unique solution $u_{\lambda, \mu}$ in $\mathcal{E}$, for any $\lambda, \mu>0$. Moreover, $u_{\lambda, \mu}$ is increasing with respect to $\lambda$ and $\mu$.

The following result give partial answers in the case where the potential $K(x)$ changes sign. 
Theorem 2.4. Assume that $K^{*}>0>K_{*}, f$ satisfies $(f 1)-(f 2)$ and $g$ verifies $(g 1)-(g 2)$. Then there exist $\lambda_{*}$ and $\mu_{*}>0$ such that problem (2.1) has at least one solution $u_{\lambda, \mu} \in \mathcal{E}$, provided that either $\lambda>\lambda_{*}$ or $\mu>\mu_{*}$. Moreover, for $\lambda>\lambda_{*}$ or $\mu>\mu_{*}, u_{\lambda, \mu}$ is increasing with respect to $\lambda$ and $\mu$.

The proofs of the above results rely on the sub- and super-solution method for elliptic equations combined with adequate comparison principles. We refer to 8] for complete details and additional results.

A natural question is to see what happens if assumption $(f 1)$ holds true, but if $\lim _{s \rightarrow \infty} f(x, s) / s$ is not zero. We give in what follows a precise description in the case where $K \leq 0$. More exactly, we consider the problem

$$
\begin{cases}-\Delta u=\lambda f(u)+a(x) g(u) & \text { in } \Omega, \\ u>0 & \text { in } \Omega, \\ u=0 & \text { on } \partial \Omega,\end{cases}
$$

where $a \in C^{0, \gamma}(\bar{\Omega}), a \geq 0, a \neq \equiv$ in $\bar{\Omega}$, and

(f3) $\quad \lim _{s \rightarrow \infty} \frac{f(s)}{s}=m \in(0, \infty)$.

Let $\lambda_{1}$ be the first Dirichlet eigenvalue of $(-\Delta)$ in $\Omega$ and $\lambda^{*}:=\lambda_{1} / m$. Set $a_{*}:=\min _{x \in \bar{\Omega}} a(x)$ and $d(x):=\operatorname{dist}(x, \partial \Omega)$.

Theorem 2.5. Assume that conditions $(f 1),(f 3),(g 1)$, and $(g 2)$ are fulfilled. Then the following hold.

(i) If $\lambda \geq \lambda^{*}$, then problem (2.2) has no solutions in $\mathcal{E}$.

(ii) If $a_{*}>0$ (resp. $a_{*}=0$ ) then problem (2.2) has a unique solution $u_{\lambda} \in \mathcal{E}$ for all $-\infty<\lambda<\lambda^{*}$ (resp. $0<\lambda<\lambda^{*}$ ) with the properties:

(ii1) $u_{\lambda}$ is strictly increasing with respect to $\lambda$;

(ii2) there exist two positive constants $c_{1}, c_{2}>0$ depending on $\lambda$ such that $c_{1} d(x) \leq u_{\lambda}(x) \leq c_{2} d(x)$, for all $x \in \Omega$;

(ii3) $\lim _{\lambda \nearrow \lambda^{*}} u_{\lambda}=+\infty$, uniformly on compact subsets of $\Omega$.

Proof. The first part of the proof relies on standard arguments based on the maximum principle (see 3] for details). The most interesting part of the proof concerns (ii3) and, due to the special character of our problem, we will be able to show that, in this case, $L^{2}$-boundedness implies $H_{0}^{1}$-boundedness! We refer to [14] for a related problem and further results.

Let $u_{\lambda} \in \mathcal{E}$ be the unique solution of (2.2) for $0<\lambda<\lambda^{*}$. We prove that $\lim _{\lambda \nearrow \lambda^{*}} u_{\lambda}=+\infty$, uniformly on compact subsets of $\Omega$. Suppose the contrary. Since $\left(u_{\lambda}\right)_{0<\lambda<\lambda^{*}}$ is a sequence of nonnegative super-harmonic functions in $\Omega$ then, by Theorem 4.1 .9 in [12], there exists a subsequence of $\left(u_{\lambda}\right)_{\lambda<\lambda^{*}}$ [still denoted by $\left.\left(u_{\lambda}\right)_{\lambda<\lambda^{*}}\right]$ which is convergent in $L_{\text {loc }}^{1}(\Omega)$.

We first prove that $\left(u_{\lambda}\right)_{\lambda<\lambda^{*}}$ is bounded in $L^{2}(\Omega)$. We argue by contradiction. Suppose that $\left(u_{\lambda}\right)_{\lambda<\lambda^{*}}$ is not bounded in $L^{2}(\Omega)$. Thus, passing eventually at a 
subsequence we have $u_{\lambda}=M(\lambda) w_{\lambda}$, where

$$
M(\lambda)=\left\|u_{\lambda}\right\|_{L^{2}(\Omega)} \rightarrow \infty \quad \text { as } \lambda \nearrow \lambda^{*} \text { and } w_{\lambda} \in L^{2}(\Omega),\left\|w_{\lambda}\right\|_{L^{2}(\Omega)}=1 .
$$

Using $(f 1),(g 2)$ and the monotonicity assumption on $g$, we deduce the existence of $A, B, C, D>0(A>m)$ such that

$$
f(t) \leq A t+B, \quad g(t) \leq C t^{-\alpha}+D, \quad \text { for all } t>0 .
$$

This implies

$$
\frac{1}{M(\lambda)}\left(\lambda f\left(u_{\lambda}\right)+a(x) g\left(u_{\lambda}\right)\right) \rightarrow 0 \quad \text { in } L_{\mathrm{loc}}^{1}(\Omega) \text { as } \lambda \nearrow \lambda^{*}
$$

that is,

$$
-\Delta w_{\lambda} \rightarrow 0 \text { in } L_{\mathrm{loc}}^{1}(\Omega) \text { as } \lambda \nearrow \lambda^{*}
$$

By Green's first identity, we have

$$
\int_{\Omega} \nabla w_{\lambda} \cdot \nabla \phi d x=-\int_{\Omega} \phi \Delta w_{\lambda} d x=-\int_{\operatorname{Supp} \phi} \phi \Delta w_{\lambda} d x \quad \forall \phi \in C_{0}^{\infty}(\Omega) .
$$

Using (2.5) we derive that

$$
\begin{aligned}
\left|\int_{\operatorname{Supp} \phi} \phi \Delta w_{\lambda} d x\right| & \leq \int_{\operatorname{Supp} \phi}|\phi|\left|\Delta w_{\lambda}\right| d x \\
& \leq\|\phi\|_{L^{\infty}} \int_{\operatorname{Supp} \phi}\left|\Delta w_{\lambda}\right| d x \rightarrow 0 \quad \text { as } \lambda \nearrow \lambda^{*}
\end{aligned}
$$

Combining (2.6) and (2.7), we arrive at

$$
\int_{\Omega} \nabla w_{\lambda} \cdot \nabla \phi d x \rightarrow 0 \text { as } \lambda \nearrow \lambda^{*}, \quad \forall \phi \in C_{0}^{\infty}(\Omega) .
$$

By definition, the sequence $\left(w_{\lambda}\right)_{0<\lambda<\lambda^{*}}$ is bounded in $L^{2}(\Omega)$.

We claim that $\left(w_{\lambda}\right)_{\lambda<\lambda^{*}}$ is bounded in $H_{0}^{1}(\Omega)$. Indeed, using (2.4) and Hölder's inequality, we have

$$
\begin{aligned}
\int_{\Omega}\left|\nabla w_{\lambda}\right|^{2} & =-\int_{\Omega} w_{\lambda} \Delta w_{\lambda}=\frac{-1}{M(\lambda)} \int_{\Omega} w_{\lambda} \Delta u_{\lambda} \\
& =\frac{1}{M(\lambda)} \int_{\Omega}\left[\lambda w_{\lambda} f\left(u_{\lambda}\right)+a(x) g\left(u_{\lambda}\right) w_{\lambda}\right] \\
& \leq \frac{\lambda}{M(\lambda)} \int_{\Omega} w_{\lambda}\left(A u_{\lambda}+B\right)+\frac{\|a\|_{\infty}}{M(\lambda)} \int_{\Omega} w_{\lambda}\left(C u_{\lambda}^{-\alpha}+D\right) \\
& =\lambda A \int_{\Omega} w_{\lambda}^{2}+\frac{\|a\|_{\infty} C}{M(\lambda)^{1+\alpha}} \int_{\Omega} w_{\lambda}^{1-\alpha}+\frac{\lambda B+\|a\|_{\infty} D}{M(\lambda)} \int_{\Omega} w_{\lambda} \\
& \leq \lambda^{*} A+\frac{\|a\|_{\infty} C}{M(\lambda)^{1+\alpha}}|\Omega|^{(1+\alpha) / 2}+\frac{\lambda B+\|a\|_{\infty} D}{M(\lambda)}|\Omega|^{1 / 2}
\end{aligned}
$$


From the above estimates, it is easy to see that $\left(w_{\lambda}\right)_{\lambda<\lambda^{*}}$ is bounded in $H_{0}^{1}(\Omega)$, so the claim is proved. Then, there exists $w \in H_{0}^{1}(\Omega)$ such that (up to a subsequence)

$$
w_{\lambda} \rightarrow w \quad \text { weakly in } H_{0}^{1}(\Omega) \text { as } \lambda \nearrow \lambda^{*}
$$

and, since $H_{0}^{1}(\Omega)$ is compactly embedded in $L^{2}(\Omega)$,

$$
w_{\lambda} \rightarrow w \quad \text { strongly in } L^{2}(\Omega) \text { as } \lambda \nearrow \lambda^{*} .
$$

On the one hand, by (2.3) and (2.10), we derive that $\|w\|_{L^{2}(\Omega)}=1$. Furthermore, using (2.8) and (2.9), we infer that

$$
\int_{\Omega} \nabla w \cdot \nabla \phi d x=0, \quad \text { for all } \phi \in C_{0}^{\infty}(\Omega) .
$$

Since $w \in H_{0}^{1}(\Omega)$, using the above relation and the definition of $H_{0}^{1}(\Omega)$, we get $w=0$. This contradiction shows that $\left(u_{\lambda}\right)_{\lambda<\lambda^{*}}$ is bounded in $L^{2}(\Omega)$. As above for $w_{\lambda}$, we can derive that $u_{\lambda}$ is bounded in $H_{0}^{1}(\Omega)$. So, there exists $u^{*} \in H_{0}^{1}(\Omega)$ such that, up to a subsequence,

$$
\begin{cases}u_{\lambda} \rightarrow u^{*} & \text { weakly in } H_{0}^{1}(\Omega) \text { as } \lambda \nearrow \lambda^{*} \\ u_{\lambda} \rightarrow u^{*} & \text { strongly in } L^{2}(\Omega) \text { as } \lambda \nearrow \lambda^{*} \\ u_{\lambda} \rightarrow u^{*} & \text { a.e. in } \Omega \text { as } \lambda \nearrow \lambda^{*}\end{cases}
$$

Now we can proceed to obtain a contradiction. Multiplying by $\varphi_{1}$ in (2.2) and integrating over $\Omega$ we have

$$
-\int_{\Omega} \varphi_{1} \Delta u_{\lambda}=\lambda \int_{\Omega} f\left(u_{\lambda}\right) \varphi_{1}+\int_{\Omega} a(x) g\left(u_{\lambda}\right) \varphi_{1}, \quad \text { for all } 0<\lambda<\lambda^{*} .
$$

On the other hand, by $(f 1)$ it follows that $f\left(u_{\lambda}\right) \geq m u_{\lambda}$ in $\Omega$, for all $0<\lambda<\lambda^{*}$. Combining this with (2.12) we obtain

$$
\lambda_{1} \int_{\Omega} u_{\lambda} \varphi_{1} \geq \lambda m \int_{\Omega} u_{\lambda} \varphi_{1}+\int_{\Omega} a(x) g\left(u_{\lambda}\right) \varphi_{1}, \quad \text { for all } 0<\lambda<\lambda^{*} .
$$

Notice that by $(g 1)$, 2.11) and the monotonicity of $u_{\lambda}$ with respect to $\lambda$ we can apply the Lebesgue convergence theorem to find

$$
\int_{\Omega} a(x) g\left(u_{\lambda}\right) \varphi_{1} d x \rightarrow \int_{\Omega} a(x) g\left(u^{*}\right) \varphi_{1} d x \text { as } \lambda \nearrow \lambda_{1} .
$$

Passing to the limit in (2.13) as $\lambda \nearrow \lambda^{*}$, and using (2.11), we obtain

$$
\lambda_{1} \int_{\Omega} u^{*} \varphi_{1} \geq \lambda_{1} \int_{\Omega} u^{*} \varphi_{1}+\int_{\Omega} a(x) g\left(u^{*}\right) \varphi_{1} .
$$

Hence $\int_{\Omega} a(x) g\left(u^{*}\right) \varphi_{1}=0$, which is a contradiction. Therefore $\lim _{\lambda \nearrow \lambda^{*}} u_{\lambda}=+\infty$, uniformly on compact subsets of $\Omega$. This concludes the proof. 


\section{Bifurcation and Asymptotics for a Singular Elliptic Equation with Convection Term}

Problems of this type arise in the study of non-Newtonian fluids, boundary layer phenomena for viscous fluids, chemical heterogeneous catalysts, cellular automata and interacting particle systems with self-organized criticality, as well as in the theory of Van der Waals interactions in thin films spreading on solid surfaces (see, e.g., [2, 6, 15]).

We are concerned in this section with singular elliptic problems of the following type

$$
\begin{cases}-\Delta u=g(u)+\lambda|\nabla u|^{p}+\mu f(x, u) & \text { in } \Omega, \\ u>0 & \text { in } \Omega, \\ u=0 & \text { on } \partial \Omega,\end{cases}
$$

where $\Omega \subset \mathbb{R}^{N}(N \geq 2)$ is a bounded domain with smooth boundary, $0<p \leq 2$, and $\lambda, \mu \geq 0$. We suppose that $f: \bar{\Omega} \times[0, \infty) \rightarrow[0, \infty)$ is a Hölder continuous function which is non-decreasing with respect to the second variable and is positive on $\bar{\Omega} \times(0, \infty)$. We assume that $g:(0, \infty) \rightarrow(0, \infty)$ is a Hölder continuous function which is non-increasing and $\lim _{s \searrow 0} g(s)=+\infty$. As in the previous section, we denote by $\lambda_{1}$ the first eigenvalue of $(-\Delta)$ in $H_{0}^{1}(\Omega)$. By the monotony of $g$, there exists $a:=\lim _{s \rightarrow \infty} g(s) \in[0, \infty)$.

The next result concerns the case $\lambda=1$ and $1<p \leq 2$.

Theorem 3.1. Assume $\lambda=1$ and $1<p \leq 2$. Then the following properties hold true.

(i) If $p=2$ and $a \geq \lambda_{1}$, then problem 3.1 has no solutions.

(ii) If either $\left[p=2\right.$ and $\left.a<\lambda_{1}\right]$ or if $1<p<2$, then there exists $\mu^{*}>0$ such that problem (3.1) has at least one classical solution for $\mu<\mu^{*}$ and no solutions exist if $\mu>\mu^{*}$.

In what follows the asymptotic behaviour of the nonlinear smooth term $f(x, u)$ will play a decisive role. We impose the following assumptions:

(f4) there exists $c>0$ such that $f(x, s) \geq c s$ for all $(x, s) \in \bar{\Omega} \times[0, \infty)$;

(f5) the mapping $(0, \infty) \ni s \longmapsto f(x, s) / s$ is non-decreasing for all $x \in \bar{\Omega}$;

(f6) the mapping $(0, \infty) \ni s \longmapsto f(x, s) / s$ is non-increasing for all $x \in \bar{\Omega}$;

(f7) $\quad \lim _{s \rightarrow \infty} f(x, s) / s=0$, uniformly for $x \in \bar{\Omega}$.

We first consider the case $\lambda=1$ and $0<p \leq 1$.

Theorem 3.2. Assume $\lambda=1$ and $0<p \leq 1$. Then the following properties hold true.

(i) If $f$ satisfies either $(f 4)$ or $(f 5)$, then there exists $\mu^{*}>0$ such that problem (3.1) has at least one classical solution for $\mu<\mu^{*}$ and no solutions exist if $\mu>\mu^{*}$.

(ii) If $0<p<1$ and $f$ satisfies $(f 6)-(f 7)$, then problem (3.1) has at least one solution for all $\mu \geq 0$. 
We now analyze the case $\mu=1$. Our framework is related to the sublinear case, described by assumptions $(f 6)$ and $(f 7)$.

Theorem 3.3. Assume $\mu=1$ and $f$ satisfies assumptions $(f 6)$ and $(f 7)$. Then the following properties hold true.

(i) If $0<p<1$, then problem (3.1) has at least one classical solution for all $\lambda \geq 0$.

(ii) If $1 \leq p \leq 2$, then there exists $\lambda^{*} \in(0, \infty]$ such that problem (3.1) has at least one classical solution for $\lambda<\lambda^{*}$ and no solution exists if $\lambda>\lambda^{*}$. Moreover, if $1<p \leq 2$, then $\lambda^{*}$ is finite.

Related to the above result we raise the following open problem: if $p=1$ and $\mu=1$, is $\lambda^{*}$ a finite number?

Theorem 3.3 shows the importance of the convection term $\lambda|\nabla u|^{p}$ in the singular problem (3.1). Indeed, according to Theorem 2.3 and for any $\mu>0$, the boundary value problem

$$
\begin{cases}-\Delta u=u^{-\alpha}+\lambda|\nabla u|^{p}+\mu u^{\beta} & \text { in } \Omega, \\ u>0 & \text { in } \Omega, \\ u=0 & \text { on } \partial \Omega\end{cases}
$$

has a unique solution, provided that $\lambda=0$ and $\alpha, \beta \in(0,1)$. Theorem 3.3 shows that if $\lambda$ is not necessarily 0 , then the following situations may occur : (i) problem (3.2) has solutions if $p \in(0,1)$ and for all $\lambda \geq 0$; (ii) if $p \in(1,2)$ then there exists $\lambda^{*}>0$ such that problem (3.2) has a solution for any $\lambda<\lambda^{*}$ and no solution exists if $\lambda>\lambda^{*}$.

We give in what follows a complete description in the special case $f \equiv 1$ and $p=2$. More precisely, we consider the problem

$$
\begin{cases}-\Delta u=g(u)+\lambda|\nabla u|^{2}+\mu & \text { in } \Omega, \\ u>0 & \text { in } \Omega, \\ u=0 & \text { on } \partial \Omega .\end{cases}
$$

A key role in this case will be played by the asymptotic behaviour of the singular term $g$. In the statement of the next result we remark some similarities with Theorem 2.5.

Theorem 3.4. The following properties hold true.

(i) Problem (3.3) has solution if and only if $\lambda(a+\mu)<\lambda_{1}$.

(ii) Assume $\mu>0$ is fixed, $g$ is decreasing and let $\lambda^{*}:=\lambda_{1} /(a+\mu)$. Then problem (3.3) has a unique solution $u_{\lambda}$ for all $\lambda<\lambda^{*}$ and the sequence $\left(u_{\lambda}\right)_{\lambda<\lambda^{*}}$ is increasing with respect to $\lambda$. Moreover, if $\lim _{\sup _{s} \backslash 0} s^{\alpha} g(s)<+\infty$, for some $\alpha \in(0,1)$, then the sequence of solutions $\left(u_{\lambda}\right)_{0<\lambda<\lambda^{*}}$ has the following properties:

(ii1) for all $0<\lambda<\lambda^{*}$ there exist two positive constants $c_{1}$, $c_{2}$ depending on $\lambda$ such that $c_{1} d(x) \leq u_{\lambda} \leq c_{2} d(x)$ in $\Omega$;

(ii2) $u_{\lambda} \in C^{1,1-\alpha}(\overline{\bar{\Omega}}) \cap C^{2}(\Omega)$;

(ii3) $u_{\lambda} \longrightarrow+\infty$ as $\lambda \nearrow \lambda^{*}$, uniformly on compact subsets of $\Omega$.

We refer to [10] for complete proofs and further details. 


\section{An Elliptic Problem with Strong Singular Nonlinearity and Convection Term}

We study the boundary value problem

$$
\begin{cases}-\Delta u=p(x) g(u)+q(x)|\nabla u|^{a} & \text { in } \Omega, \\ u>0 & \text { in } \Omega, \\ u=0 & \text { on } \partial \Omega,\end{cases}
$$

where $\Omega \subset \mathbb{R}^{N}(N \geq 2)$ is a smooth bounded domain, $0<a<1$ and $q \in C^{0, \alpha}(\bar{\Omega})$, $q>0$ in $\bar{\Omega}$. The potential $p \in C^{1}(\Omega)$ satisfies

$$
c_{1} d(x)^{\beta} \leq|p(x)| \leq c_{2} d(x)^{\beta}, \quad \text { for all } x \in \Omega,
$$

where $c_{1}, c_{2}>0$, and $\beta$ is a real number. This assumption shows that the potential $p(x)$ can admit a singular boundary behaviour (corresponding to $\beta<0$ ).

Throughout this section we suppose that $g \in C^{1}(0, \infty)$ is a positive decreasing function such that $\lim _{s \searrow 0} g(s)=+\infty$. The blow-up rate of $g$ at the origin is described by the following assumption:

(g4) there exists $\gamma>\max \{1, \beta+1\}$ such that $\lim _{s \backslash 0} s^{\gamma} g(s) \in(0, \infty)$.

Observe that the stronger decay of the singular nonlinearity $g$ around the origin [described by our assumption $(g 4)$ ] implies that $g$ does not obey the KellerOsserman type condition ( $g 3)$.

From (4.2) we deduce that $p$ does not vanish in $\Omega$. Our first result concerns the case $p<0$ in $\Omega$.

Theorem 4.1. Assume that $g$ satisfies $(g 4), p$ is negative in $\Omega$, and condition (4.2) is fulfilled. Then problem (4.1) has no classical solutions.

Proof. Let $\varphi_{1}$ be the normalized positive eigenfunction corresponding to the first eigenvalue $\lambda_{1}$ of $(-\Delta)$ in $H_{0}^{1}(\Omega)$. Then $\lambda_{1}>0, \varphi_{1} \in C^{2}(\bar{\Omega})$, and

$$
C_{1} d(x) \leq \varphi_{1}(x) \leq C_{2} d(x), \quad x \in \Omega,
$$

for some positive constants $C_{1}$ and $C_{2}$. From (4.2) and (4.3) it follows that there exist $\tau_{1}, \tau_{2}>0$ such that

$$
\tau_{1} \varphi_{1}(x)^{\beta} \leq|p(x)| \leq \tau_{2} \varphi_{1}(x)^{\beta}, \quad \text { for all } x \in \Omega .
$$

Fix $C>0$ such that $\|q\|_{\infty}^{2} C^{a-1}<\lambda_{1}$ and define $\psi:[0, \infty) \rightarrow[0, \infty)$ by $\psi(s)=s^{a} /\left(s^{2}+C\right)$. Then $\psi$ attains its maximum at $\bar{s}=[C a /(2-a)]^{1 / 2}$. Hence

$$
\psi(s) \leq \psi(\bar{s})=\frac{a^{a / 2}(2-a)^{(2-a) / 2}}{2 C^{1-a / 2}}, \quad \text { for all } s \geq 0 .
$$

An elementary computation shows that

$$
s^{a} \leq C^{a / 2-1} s^{2}+C^{a / 2}, \quad \text { for all } s \geq 0 .
$$


Arguing by contradiction, let us assume that problem (4.1) has a classical solution $U$. Consider the perturbed problem

$$
\begin{cases}-\Delta u=p(x) g(u+\varepsilon)+A|\nabla u|^{2}+B & \text { in } \Omega \\ u>0 & \text { in } \Omega \\ u=0 & \text { on } \partial \Omega\end{cases}
$$

where $\varepsilon>0$, and $A=\|q\|_{\infty} C^{a / 2-1}, B=\|q\|_{\infty} C^{a / 2}$. By virtue of (4.5) it follows that $U$ is a sub-solution of (4.6). Set $v=e^{A u}-1$. Then problem (4.6) becomes

$$
\begin{cases}-\Delta v=A p(x)(v+1) g\left(\frac{1}{A} \ln (v+1)+\varepsilon\right)+A B(v+1) & \text { in } \Omega, \\ v>0 & \text { in } \Omega, \\ v=0 & \text { on } \partial \Omega .\end{cases}
$$

We first remark that $V=e^{A U}-1$ is a sub-solution of (4.7). On the other hand, since $A B<\lambda_{1}$, we conclude that there exists $w \in C^{2}(\bar{\Omega})$ such that

$$
\begin{cases}-\Delta w=A B(w+1) & \text { in } \Omega, \\ w>0 & \text { in } \Omega, \\ w=0 & \text { on } \partial \Omega .\end{cases}
$$

Moreover, the maximum principle yields

$$
c_{1} \varphi_{1} \leq w \leq c_{2} \varphi_{2} \quad \text { in } \Omega,
$$

for some positive constants $c_{1}$ and $c_{2}>0$. It is clear that $w$ is a super-solution of (4.7). We claim that $V \leq w$. To this aim, it suffices to prove that $U \leq W$ in $\Omega$, where $W=A^{-1} \ln (w+1)$ verifies

$$
\begin{cases}-\Delta W=A|\nabla W|^{2}+B & \text { in } \Omega \\ W>0 & \text { in } \Omega, \\ W=0 & \text { on } \partial \Omega .\end{cases}
$$

Assuming the contrary, we get that $\max _{x \in \bar{\Omega}}(U-W)>0$ is achieved in some point $x_{0} \in \Omega$. Then $\nabla(U-W)\left(x_{0}\right)=0$ and

$0 \leq-\Delta(U-W)\left(x_{0}\right)=p\left(x_{0}\right) g\left(U\left(x_{0}\right)\right)+q\left(x_{0}\right)|\nabla U|^{a}\left(x_{0}\right)-A|\nabla W|^{2}\left(x_{0}\right)-B<0$, which is a contradiction. Hence $U \leq W$ in $\Omega$, that is, $V \leq w$ in $\Omega$. By the sub and super-solution method we deduce that there exists $v_{\varepsilon} \in C^{2}(\bar{\Omega})$ a solution of problem (4.7) such that

$$
V \leq v_{\varepsilon} \leq w \quad \text { in } \Omega .
$$

Now we proceed to get our contradiction. Integrating in (4.7) and taking into account the fact that $p$ is negative, we deduce

$$
-\int_{\Omega} \Delta v_{\varepsilon} d x-A \int_{\Omega} p(x) g\left(\frac{1}{A} \ln \left(v_{\varepsilon}+1\right)+\varepsilon\right) d x \leq A B \int_{\Omega}(w+1) d x .
$$

Using monotonicity of $g$ and the fact that $\ln \left(v_{\varepsilon}+1\right) \leq v_{\varepsilon}$ in $\Omega$, the above inequality yields

$$
-\int_{\partial \Omega} \frac{\partial v_{\varepsilon}}{\partial n} d s-A \int_{\Omega} p(x) g\left(\frac{v_{\varepsilon}}{A}+\varepsilon\right) d x \leq A B\left(\|w\|_{\infty}+1\right)|\Omega|<+\infty .
$$


Since $\partial v_{\varepsilon} / \partial n \leq 0$ on $\partial \Omega$, the above relation implies

$$
-\int_{\Omega} p(x) g\left(\frac{v_{\varepsilon}}{A}+\varepsilon\right) d x \leq M,
$$

where $M=B\left(\|w\|_{\infty}+1\right)|\Omega|$. Now, relations (4.10) and (4.11) imply

$$
0 \leq-\int_{\Omega} p(x) g\left(\frac{w}{A}+\varepsilon\right) d x \leq M .
$$

Therefore, for any compact subset $\omega \subset \subset \Omega$ we have

$$
0 \leq-\int_{\omega} p(x) g\left(\frac{w}{A}+\varepsilon\right) d x \leq M .
$$

Passing to the limit as $\varepsilon \searrow 0$ in the above inequality, it follows that

$$
-\int_{\omega} p(x) g\left(\frac{w}{A}\right) d x \leq M, \quad \text { for all } \omega \subset \subset \Omega .
$$

This yields

$$
-\int_{\Omega} p(x) g\left(\frac{w}{A}\right) d x \leq M .
$$

On the other hand, the hypothesis ( $g 4)$ combined with (4.9) implies $g(w / A) \geq$ $c_{0} \varphi_{1}^{-\gamma}$ in $\Omega$, for some $c_{0}>0$. The last inequality together with (4.4) and 4.12) produces $c \int_{\Omega} \varphi_{1}^{\beta-\gamma} d x \leq M$, where $\beta-\gamma<-1$. But, by a result of Lazer and McKenna (see [13]), $\int_{\Omega} \varphi_{1}^{-s} d x<+\infty$ if and only if $s<1$. This contradiction shows that problem (4.1) has no classical solutions and the proof is now complete.

The situation changes radically in the case where $p$ is positive in $\Omega$, as established in the next result.

Theorem 4.2. Assume that $g$ satisfies $(g 4)$ and the potential $p(x)$ is positive and fulfills (4.2). Then the following properties hold true.

(i) If $\beta \leq-2$, then problem (4.1) has no classical solutions.

(ii) If $\beta>-2$, then problem (4.1) has a unique solution $u$ which, moreover, has the following properties:

(ii1) there exist $M, m>0$ such that

$$
m d(x)^{(2+\beta) /(1+\gamma)} \leq u(x) \leq M d(x)^{(2+\beta) /(1+\gamma)}, \quad \text { for all } x \in \Omega ;
$$

(ii2) if $\beta \geq \max \{0, \gamma-3\}$, then $u$ is in $H_{0}^{1}(\Omega)$;

(ii3) if $2 \beta \leq \gamma-3$, then $u$ does not belong to $H_{0}^{1}(\Omega)$.

We refer to 11$]$ for the proof of Theorem 4.2 as well as for a result concerning the entire solutions of problem (4.1). 


\section{References}

[1] P. Bénilan, H. Brezis, and M. Crandall, A semilinear equation in $L^{1}\left(\mathbb{R}^{N}\right)$. Ann. Scuola Norm. Sup. Pisa 4 (1975), 523-555.

[2] J. T. Chayes, S. J. Osher, and J. V. Ralston, On singular diffusion equations with applications to self-organized criticality. Comm. Pure Appl. Math. 46 (1993), 13631377.

[3] F.-C. Cîrstea, M. Ghergu, and V. Rădulescu, Combined effects of asymptotically linear and singular nonlinearities in bifurcation problems of Lane-Emden-Fowler type. J. Math. Pures Appl., in press.

[4] M. Coclite and G. Palmieri, On a singular nonlinear Dirichlet problem. Commun. Partial Diff. Equations 14 (1989), 1315-1327.

[5] M. G. Crandall, P. H. Rabinowitz, and L. Tartar, On a Dirichlet problem with a singular nonlinearity. Commun. Partial Diff. Equations 2 (1977), 193-222.

[6] P. G. de Gennes, Wetting: statics and dynamics. Review of Modern Physics 57 (1985), $827 \mathrm{x}-863$.

[7] M. Ghergu and V. Rădulescu, Bifurcation and asymptotics for the Lane-EmdenFowler equation. C. R. Acad. Sci. Paris, Ser. I 337 (2003), 259-264.

[8] M. Ghergu and V. Rădulescu, Sublinear singular elliptic problems with two parameters. J. Differential Equations 195 (2003), 520-536.

[9] M. Ghergu and V. Rădulescu, Bifurcation for a class of singular elliptic problems with quadratic convection term. C. R. Acad. Sci. Paris, Ser. I 338 (2004), 831-836.

[10] M. Ghergu and V. Rădulescu, Multiparameter bifurcation and asymptotics for the singular Lane-Emden-Fowler equation with a convection term. Proc. Royal Soc. Edinburgh Sect. A, in press.

[11] M. Ghergu and V. Rădulescu, Singular elliptic problems with sublinear convection term and Kato potential in anisotropic media, in preparation.

[12] L. Hörmander, The Analysis of Linear Partial Differential Operators I. Springer, Berlin, 1983.

[13] A. C. Lazer and P. J. McKenna, On a singular nonlinear elliptic boundary value problem. Proc. Amer. Math. Soc. 3 (1991), 720-730.

[14] P. Mironescu and V. Rădulescu, The study of a bifurcation problem associated to an asymptotically linear function. Nonlinear Anal., T.M.A. 26 (1996), 857-875.

[15] J. Ockendon, S. Howison, A. Lacey, and A. Movchan, Applied Partial Differential Equations, Oxford University Press, 2003.

[16] J. Shi and M. Yao, On a singular nonlinear semilinear elliptic problem. Proc. Roy. Soc. Edinburgh Sect. A 128 (1998), 1389-1401.

\section{Acknowledgment}

I am greatly indebted to Professor Haim Brezis, for his highest level guidance during my $\mathrm{PhD}$ and Habilitation theses at the Université Pierre et Marie Curie (Paris 6), as well as for suggesting to me several modern research subjects and directions of interest. Bonne anniversaire, mon Professeur! 
Vicenţiu Rădulescu

Department of Mathematics

University of Craiova

RO 200585 Craiova

Romania

http://inf.ucv.ro/ ªdulescu

e-mail: radulescu@inf .ucv.ro 